Rong Shan et al., PdLuBi

\title{
Electronic and crystalline structures of zero band-gap PdLuBi thin films grown epitaxially on $\mathrm{MgO}(100)$
}

\author{
Rong Shan, ${ }^{1,2}$ Siham Ouardi, ${ }^{3}$ Gerhard H. Fecher, ${ }^{3}$ Li Gao, ${ }^{2}$ Andrew Kellock, ${ }^{2}$ Kevin P. Roche, ${ }^{2}$ Mahesh G. \\ Samant, ${ }^{2}$ Carlos E. Vidal Barbosa, ${ }^{3}$ Eiji Ikenaga, ${ }^{4}$ Claudia Felser, ${ }^{3}$ and Stuart S. P. Parkin ${ }^{2, a)}$ \\ ${ }^{1)}$ Institut für Anorganische und Analytische Chemie, Johannes Gutenberg - Universität, 55099 Mainz, \\ Germany. \\ ${ }^{2)}$ IBM Almaden Research Center San Jose, CA 95120, USA. \\ 3) Max Planck Institute for Chemical Physics of Solids, 01187 Dresden, Germany. \\ 4) Japan Synchrotron Radiation Research Institute (JASRI), SPring-8, Hyogo 679-5198, \\ Japan.
}

(Dated: 31 October 2018)

Thin films of the proposed topological insulator PdLuBi - a Heusler compound with the $C 1_{b}$ structure - were prepared on Ta-Mo-buffered $\mathrm{MgO}(100)$ substrates by co-sputtering from $\mathrm{PdBi}_{2}$ and $\mathrm{Lu}$ targets. Epitaxial growth of high-quality PdLuBi films was confirmed by X-ray spectrometry and reflection high-energy electron diffraction. The root-mean-square roughness of the films was as low as $1.45 \mathrm{~nm}$, although the films were deposited at high temperature. The film composition is close to the ideal stoichiometric ratio. The valence band spectra of the PdLuBi films, observed by hard X-ray photoelectron spectroscopy, correspond perfectly to the ab-initio-calculated density of states.

Keywords: Heusler compounds, Thin films, Topological insulators, Electronic structure, Photoelectron spectroscopy

Zero energy consumption in electron transport, spin channel splitting, protection of spins from thermal activity, and other interesting, recently proposed, physical properties in topological insulators (TIs) would greatly improve the performance of semiconductor devices, achieve spin control in spintronics devices without application of magnetic fields, and provide an excellent platform for the application of quantum physics, e.g., quantum computers ${ }^{1-4}$. Until now, most studies of TIs have focused on $\mathrm{HgTe} / \mathrm{CdTe} / \mathrm{HgTe}$ quantum wells with two-dimensional (2D) topological states ${ }^{4}$ and three-dimensional (3D) TIs, $\mathrm{Bi}_{1-x} \mathrm{Sb}_{x}, \mathrm{Bi}_{1-x} \mathrm{Te}_{x}$, and $\mathrm{Sb}_{1-x} \mathrm{Te}_{x}{ }^{5-7}$. Electrical properties related to the quantum spin Hall effect have only been observed in $\mathrm{HgTe} / \mathrm{CdTe} / \mathrm{HgTe}$ quantum wells, which have been fabricated by molecular beam epitaxy and used as rudimentary devices. Even for $\mathrm{HgTe} / \mathrm{CdTe} / \mathrm{HgTe}$ quantum wells, the difficult and expensive fabrication of the devices has impeded further study. In order to resolve this problem, we suggest a new platform based on half-Heusler compounds for the study of $\mathrm{TIs}^{8}$. A band gap arises in half-Heusler compounds when the total number of valence electrons equals 18 as a result of a closed-shell electronic configuration ${ }^{9}$, although the material consists of three metals. The $\Gamma_{6}$ and $\Gamma_{8}$ bands are inverted in some semiconducting Heusler compounds with heavy elements as a result of strong spin-orbit coupling ${ }^{8,10}$. This leads to a TI state with a zero band gap that can be used in 2D TI quantum well structures. Considering the large range of lattice constants corresponding to the variety of materials and the easy fabrication conditions of Heusler com-

\footnotetext{
a)Electronic mail: Stuart.Parkin@us.ibm.com
}

pounds, we suggest that TI devices with Heusler compounds may become feasible for applications. For this purpose, high-quality epitaxial PdLuBi films were fabricated by co-sputtering.

Samples with the structures $\mathrm{MgO}(100) / \mathrm{Mo} \quad(2 \mathrm{~nm}) / \mathrm{Ta} \quad(20 \mathrm{~nm}) / \mathrm{Mo} \quad\left(\begin{array}{ll}1 & \mathrm{~nm}\end{array}\right) /$ PdLuBi $(40 \mathrm{~nm}) / \mathrm{MgO}(17 \mathrm{~nm})$ were prepared by direct-current magnetron co-sputtering under an $\mathrm{Ar}$ atmosphere. The base pressure of the sputtering chamber was below $5 \times 10^{-7} \mathrm{~Pa}$. The angle of inclination between each target and the substrate was about $9^{\circ}$ and the distance between the sources and target was about 7.95 inches. The substrate was rotated during film growth. The composition of the PdLuBi thin film was analyzed by Rutherford backscattering spectrometry and particle-induced X-ray emission. The crystalline structure was examined by X-ray diffraction (D8-Discover, Bruker AXS Inc.). The surface morphologies of the films were analyzed in situ using reflection high- energy electron diffraction (RHEED, STAIB Instrument Inc.). Ex situ surface analysis was carried out using atomic force microscopy (5600LS, Agilent Technologies Inc.). The valence band spectra of the PdLuBi films were observed using hard-X-Ray photoelectron spectroscopy (HAXPES) at BL47XU of Spring-8 (Japan). For details of the HAXPES experiments, see References ${ }^{11-13}$.

Mo and Ta layers were deposited at $200^{\circ} \mathrm{C}$, and then annealed at $900^{\circ} \mathrm{C}$. The Mo layer was used to induce growth of $\mathrm{Ta}(100)$ on $\mathrm{MgO}(100)$ instead of $\mathrm{Ta}(110)$. After this process, a single-crystalline film of $\mathrm{Ta}-\mathrm{Mo}(100)$ was built on $\mathrm{MgO}(100)$ with a $45^{\circ}$ in-plane rotation. Because Mo and Ta can be miscible with each other at high temperatures, the lattice constant of Ta-Mo can be tuned by precise adjustment of the composition. In this study, the lattice constant of PdLuBi was slightly 
smaller than twice the lattice constant of Ta. A thin Mo layer was therefore used between the Ta and PdLuBi layers. The root-mean-square roughness of the $\mathrm{Ta}-\mathrm{Mo}$ layer was below $3 \AA$. The PdLuBi layer was deposited at $800^{\circ} \mathrm{C}$. To obtain a semiconducting Heusler film of high quality, a high substrate temperature is usually necessary. However, as a result of the low bonding energy between $\mathrm{Bi}$ atoms, Bi almost disappears above a substrate temperature of $600^{\circ} \mathrm{C}$ when the film is sputtered from three separate targets. On the other hand, the melting point of $\mathrm{PdBi}_{2}\left(\approx 480^{\circ} \mathrm{C}\right)$ is much higher than that of $\mathrm{Bi}$ $\left(\approx 271^{\circ} \mathrm{C}\right.$ ), implying a higher bonding energy of $\mathrm{Pd}-\mathrm{Bi}$. $\mathrm{Bi}$ was therefore sputtered from a $\mathrm{PdBi}_{2}$ target to reduce the problem of $\mathrm{Bi}$ loss at high growth temperatures. In this case, the composition ratio of $\mathrm{Pd}$ :Bi could be tuned using the annealing temperature and the composition ratio of Lu:Pd could be tuned using the sputtering power. Using this procedure, films with a composition ratio of Pd:Lu:Bi $=33.5: 33.4: 33.1($ at $\% ; \pm 1 \%)$ were obtained at $800^{\circ} \mathrm{C}$.

Figure 1 shows the X-ray diffraction pattern of a $\mathrm{Pd}$ LuBi thin film on Ta-Mo-buffered $\mathrm{MgO}(100)$. Only the (200) and (400) diffraction peaks of the PdLuBi film are observed in the (100) polar $2 \theta$-scan (see $1(\mathrm{a}))$. The lattice constant of the PdLuBi film is $\approx 6.56 \AA$ and corresponds to that reported for the bulk material ${ }^{14}$. The (111) polar scan and the azimuth $\phi$-scan of the PdLuBi(111) diffraction peak (inset of $1(\mathrm{~b})$ ) with four-fold symmetry prove epitaxial growth of the PdLuBi film.

The surface morphology of the film is shown in Figure 2(a). The root-mean-square roughness is around $1.45 \mathrm{~nm}$, although the film was deposited at $800^{\circ} \mathrm{C}$. The RHEED pattern of the PdLuBi film without a cap layer is shown in Figure 2(b). It is known that single-crystalline films with an atomically flat 2D surface produce a stripe pattern, whereas single-crystalline films with an atomically rough $(3 \mathrm{D})$ surface produce a transmission pattern, i.e., a set of broad spots ${ }^{15}$. Polycrystalline films and textured films without in-plane orientation form a set of concentric circles instead. It is clear from Figure 2 that a spotty pattern was observed. This indicates that a single-crystalline PdLuBi film with an atomically rough surface - either via a 3D island (Volmer-Weber) growth mode or a layer-plus-island (Stranski-Krastanov) growth mode-was obtained successfully.

The electronic structure of PdLuBi was calculated using WIEN $2 \mathrm{~K}^{16}$. More details are given in Reference ${ }^{17}$. Spin-orbit interactions were included for all atoms and $\mathrm{LDA}+U$ with the self- interaction correction doublecounting scheme for Lu. An effective Coulomb energy of $U_{\text {eff }}=U-J=0.5$ Ry was used to reproduce the measured energy of the Lu $4 f$ doublet; $a=6.6787 \AA$ was found for the relaxed lattice parameter. The result of the electronic structure calculations is shown in Figure 3. The compound turns out to be similar to a zero band gap semiconductor (compare ${ }^{18,19}$ and references there). The optical gap at $\Gamma$ is closed. The valence and conduction bands have a small overlap of about $130 \mathrm{meV}$. A frac-
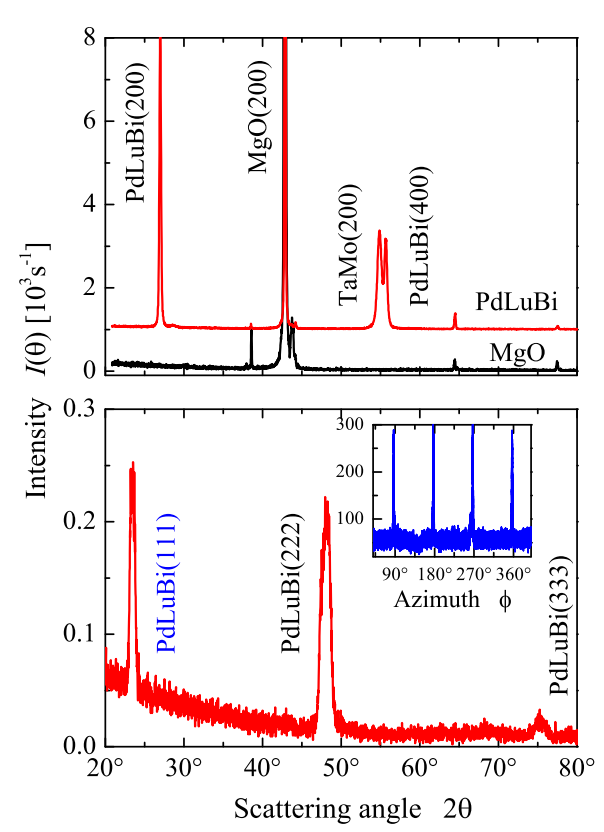

FIG. 1. (Color online) X-ray diffraction patterns of thin PdLuBi films on Ta-Mo-buffered $\mathrm{MgO}(100)$.

(a) (100) polar scan of PdLuBi (red curve); the black solid curve indicates the background from the $\mathrm{MgO}(100)$ substrate. (b) (111) polar scan of PdLuBi and azimuth scan of Pd$\operatorname{LuBi}(111)$ (inset).

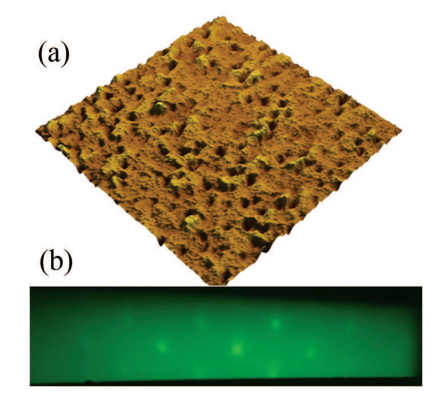

FIG. 2. (Color online) Morphology of thin PdLuBi films.

(a) Atomic force microscopy three-dimensional surface representation of PdLuBi with cap layer, (b) reflection high-energy electron diffraction pattern of PdLuBi.

tion of about $10^{-3}$ electrons is found in the conduction band. This situation may change for slightly smaller lattice parameters (see Figure 3(c)) or a slight tetragonal distortion. Both effects are principally able to open up the band gap again at $\Gamma$ (compare also Reference $\left.{ }^{10}\right)$. A slight tetragonal distortion may particularly occur in thin films with pseudomorphic growth.

Valence band spectra of PdLuBi are presented in Figure 4 and compared to the calculated density of states. Figure 4(a) shows the valence band spectra excited by photons of energy about $8 \mathrm{keV}$. The high intensity with a maximum between -12.5 and $-11 \mathrm{eV}$ corresponds to excitation of the low-lying $\mathrm{Bi} s$ states with $a_{1}$ symmetry, 

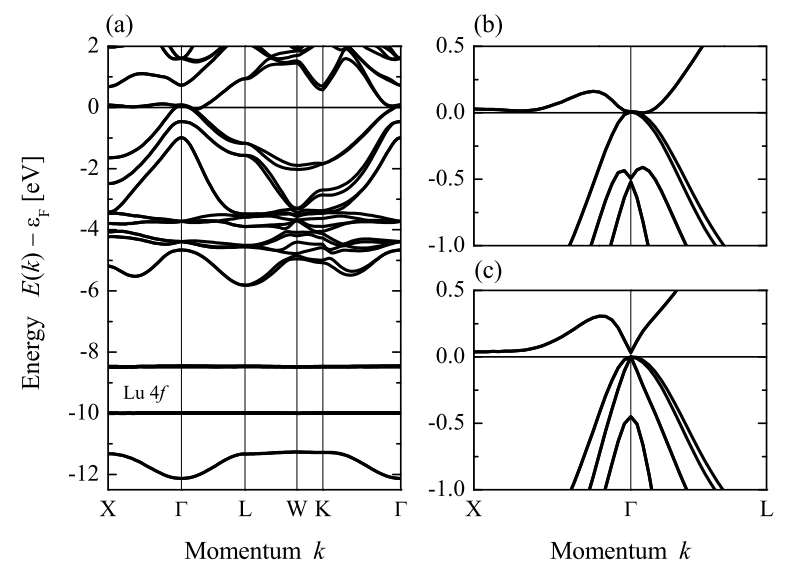

FIG. 3. Electronic structure of PdLuBi.

(a) shows the complete band structure for the relaxed lattice parameter. (b) and (c) show the band structure in the vicinity of the Fermi energy for the thin film lattice parameter and a $5 \%$ compressed lattice parameter, respectively. In (c), a small band gap of width $40 \mathrm{meV}$ is opened. Calculations were performed with spin-orbit interactions for all elements and $\mathrm{LDA}+U$ for the $\mathrm{Lu} 4 f$ electrons.

as is seen by comparison with the density of states. The $\mathrm{Lu} 4 f_{5 / 2}-4 f_{7 / 2}$ spin-orbit doublet is found at energies of $E_{5 / 2}=-9.76 \mathrm{eV}$ and $E_{7 / 2}=-8.31 \mathrm{eV}$, with a spin-orbit splitting of $\Delta_{\mathrm{SO}}=1.45 \mathrm{eV}$. The Lu $4 f$ states reside on top of the states from the $\mathrm{MgO}$ protective overlayer that usually appear at about $-9 \mathrm{eV}$. Such $\mathrm{MgO}$ states were previously also observed in the spectra from $\mathrm{MgO}$-covered $\mathrm{Co}_{2} \mathrm{MnSi}^{11}$. The upper part of the valence spectra above $-6.5 \mathrm{eV}$ exhibits the typical structure of the PdLuBi valence $d$ bands with four major maxima $(-6,-5.16,-4.31$, $-2.1 \mathrm{eV}$ ) and a smaller one at $-2.9 \mathrm{eV}$. The splitting of the states at about $-4 \mathrm{eV}$ is not resolved in the spectrum. Slight energy differences between the maxima in the density of states and maxima of the spectrum are observed. These energy shifts are larger for states further away from the Fermi energy (for example $0.3 \mathrm{eV}$ at the $-2.1 \mathrm{eV}$ maximum and $0.6 \mathrm{eV}$ at the $-5.16 \mathrm{eV}$ maximum). This is a typical effect of the photoemission process and emerges from the complex self-energy of the photoelectrons interacting with the remaining $N-1$ electron system. The spectra exhibit no clear cut-off at the Fermi energy as is observed when a metallic-type density is terminated by the Fermi-Dirac distribution, but a rather smooth, linearly decreasing intensity is observed. A similar behavior was found in bulk materials of the zero band gap Heusler compounds $\mathrm{PtYSb}^{18}$ and $\mathrm{PtLuSb}^{19}$. A splitting of the La $3 d$ states was reported for thin PtLaBi films grown on $\mathrm{YAlO}_{3}(001)$ by three-source magnetron co-sputtering ${ }^{20}$. The appearance of the satellite peaks is explained as being the result of charge transfer, as appears, for example, in oxides. The PdLuBi films reported here did not exhibit any additional splitting of core levels, which also indicates their high quality with respect to impurities,
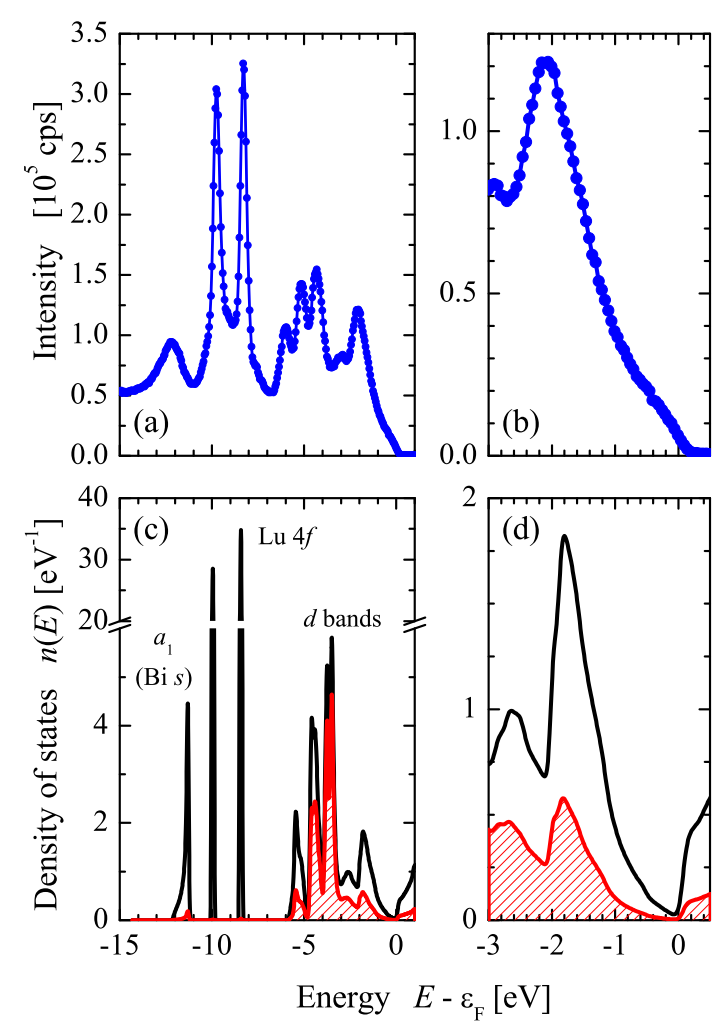

FIG. 4. (Color Online) Valence band of PdLuBi.

(a)-(b) show the valence band spectra taken with a photon energy of about $8 \mathrm{keV}$. (c)-(d) show the density of states, and the density localized at the Pd atoms is marked by the shaded area. (b) and (d) show the valence band close to the Fermi energy on an enlarged scale.

structure, and composition.

In summary, high-quality epitaxial thin films of the Heusler TI PdLuBi were successfully prepared. The composition of $\mathrm{Pd}: \mathrm{Lu}: \mathrm{Bi}$ in the film grown at $800^{\circ} \mathrm{C}$ is stoichiometric, with ratios of 1:1:1. The valence band spectra of thin PdLuBi films observed by HAXPES perfectly match the density of states from first principles calculations. PdLuBi has a similar band structure to that of $\mathrm{HgTe}$ and thus may be used for the construction of $2 \mathrm{D}$ TI quantum well structures.

\section{ACKNOWLEDGMENTS}

Financial support by the DFG-JST (projects P 1.3$\mathrm{A}$ and $\mathrm{P}$ 2.3-A in research unit FOR 1464 ASPIMATT) is gratefully acknowledged. HAXPES was performed at BL47XU of SPring-8 with approval of JASRI (Proposal No. 2012A0043).

\footnotetext{
${ }^{1}$ C. L. Kane and E. J. Mele, Phys. Rev. Lett. 95, 146802 (2005). ${ }^{2}$ B. A. Bernevig, T. L. Hughes, and S. C. Zhang, Science 314, 1757 (2006).
} 
${ }^{3}$ X. Qi, T. L. Hughes, and S. C. Zhang, Phys. Rev. B 78, 195424 (2008).

${ }^{4}$ M. Konig, S. Wiedmann, C. Brune, A. Roth, H. Buhmann, L. W. Molenkamp, X. L. Qi, and S. C. Zhang, Science 318, 766 (2007).

${ }^{5}$ L. Fu, C. L. Kane, and E. J. Mele, Phys. Rev. Lett. 98, 106803 (2007).

${ }^{6}$ H. J. Zhang, C. X. Liu, X. L. Qi, X. Dai, Z. Fang, and S. C. Zhang, Nature Phys. 5, 438 (2009).

${ }^{7}$ Y. L. Chen, J. G. Analytis, J. H. Chu, Z. K. Liu, S. K. Mo, X. L. Qi, H. J. Zhang, D. H. Lu, X. Dai, Z. Fang, S. C. Zhang, I. R. Fisher, Z. Hussain, and Z. X. Shen, Science 325, 178 (2009).

${ }^{8}$ S. Chadov, X. L. Qi, J. Kübler, G. H. Fecher, C. Felser, and S. C. Zhang, Nature Mat. 9, 541 (2010).

${ }^{9}$ D. Jung, H. J. Koo, and M. H. Whangbo, J. Mol. Struct. Theochem. 527, 113 (2000).

${ }^{10}$ D. Xiao, Y. Yao, W. Feng, J. Wen, W. Zhu, X.-Q. Chen, G. M. Stocks, and Z. Zhang, Phys. Rev. Let. 105, 096404 (2010).

${ }^{11}$ G. H. Fecher, B. Balke, A. Gloskowskii, S. Ouardi, C. Felser, T. Ishikawa, M. Yamamoto, Y. Yamashita, H. Yoshikawa, S. Ueda, and K. Kobayashi, Appl. Phys. Lett. 92, 193513 (2008).

${ }^{12}$ S. Ouardi, G. H. Fecher, X. Kozina, G. Stryganyuk, B. Balke, C. Felser, E. Ikenaga, T. Sugiyama, N. Kawamura, M. Suzuki, and K. Kobayashi, Phys. Rev. Lett. 107, 036402 (2011).

${ }^{13}$ X. Kozina, G. H. Fecher, G. Stryganyuk, S. Ouardi, B. Balke,
C. Felser, G. Schönhense, E. Ikenaga, T. Sugiyama, N. Kawamura, M. Suzuki, T. Taira, T. Uemura, M. Yamamoto, H. Sukegawa, W. Wang, K. Inomata, and K. Kobayashi, Phys. Rev. B 84, 054449 (2011).

${ }^{14}$ M. G. Hasse, T. Schmidt, C. G. Richter, H. Block, and W. Jeitschko, J. Solid State Chem. 168, 18 (2002).

${ }^{15}$ A. Ichimiya and P. I. Cohen, Reflection High Energy Electron Diffraction (Cambridge University Press, Cambridge, UK, 2004).

${ }^{16}$ P. Blaha, K. Schwarz, G. K. H. Madsen, D. Kvasnicka, and J. Luitz, WIEN2k, An Augmented Plane Wave + Local Orbitals Program for Calculating Crystal Properties (Karlheinz Schwarz, Techn. Universität Wien, Wien, Austria, 2001).

${ }^{17}$ S. Ouardi, G. H. Fecher, B. Balke, X. Kozina, G. Stryganyuk, C. Felser, S. Lowitzer, D. Ködderitzsch, H. Ebert, and E. Ikenaga, Phys. Rev. B 82, 085108 (2010).

${ }^{18}$ S. Ouardi, C. Shekhar, G. H. Fecher, X. Kozina, G. Stryganyuk, C. Felser, S. Ueda, and K. Kobayashi, Appl. Phys. Lett. 98, 211901 (2011).

${ }^{19}$ C. Shekhar, S. Ouardi, G. H. Fecher, A. K. Nayak, C. Felser, and E. Ikenaga, Appl. Phys. Lett. 100, 252109 (2012).

${ }^{20}$ T. Miyawaki, N. Sugimoto, N. Fukatani, T. Yoshihara, K. Ueda, N. Tanaka, and H. Asano, J. Appl. Phys. 111, 07E327 (2012). 